\title{
Isoparametric Boundary Elements
}

Matthias Weigelt

Geodätisches Institut Stuttgart

weigelt@gis.uni-stuttgart.de

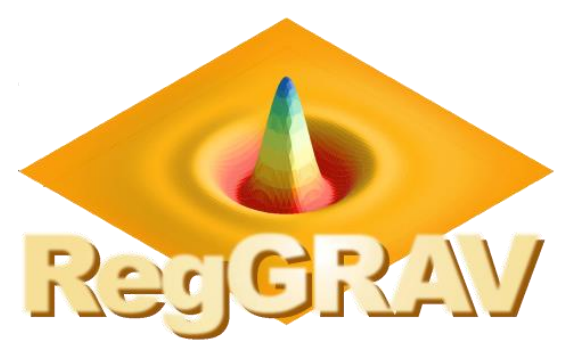

Workshop on Regional Gravity and Geomagnetic Field Modelling

Bavarian Academy of Sciences and Humanities, Munich

February 23-24, 2012 


\section{Application}

Primary objective: $\quad$ local gravity field determination from CHAMP, GRACE (and GOCE)

Interest:

improved static and time-variable gravity field (make use of residual signal and data distribution)

Input data: potential values along the orbit gravity gradient along the orbit relative gradient along the orbit

Data type: currently only single data type

However: no restrictions on the combination of different data types (combination on normal equation level) 


\section{Basic principle}

Decomposition of the surface into elements with finite extend (boundary elements)

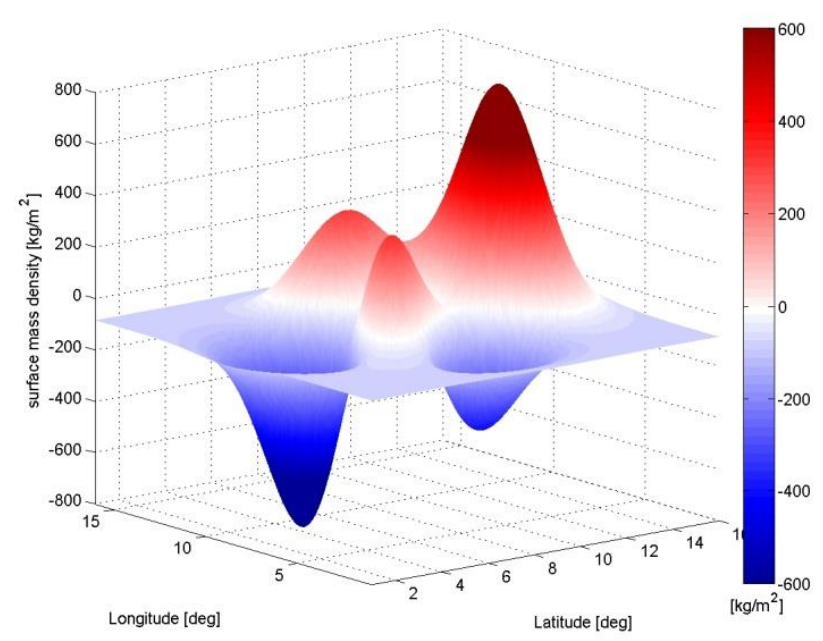

\section{RegGrav Workshop}

Feb. 23-24, 2012, Munich

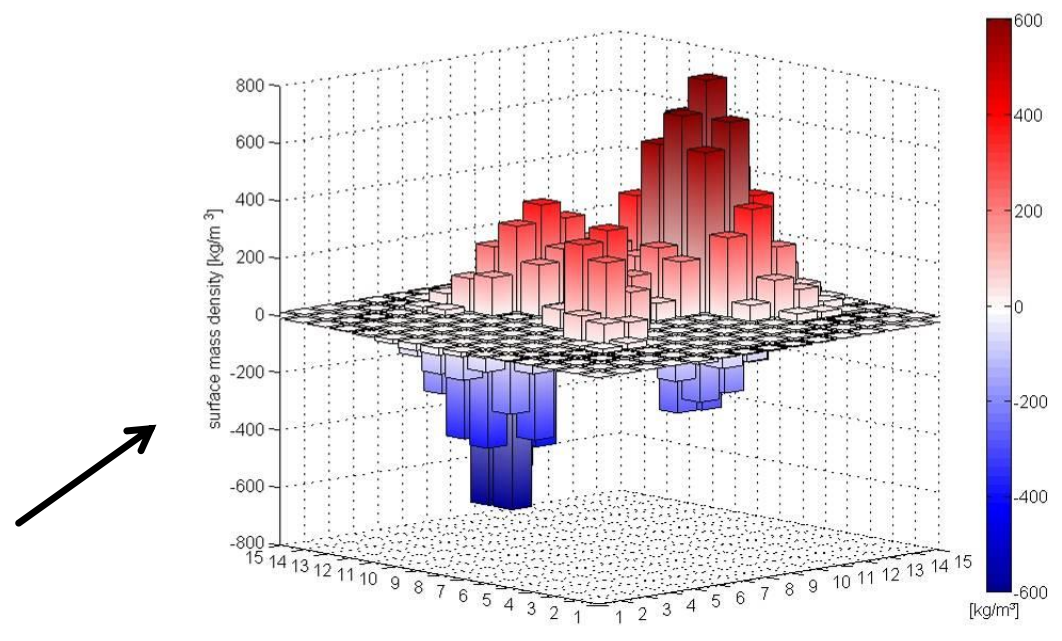

e.g. blocks
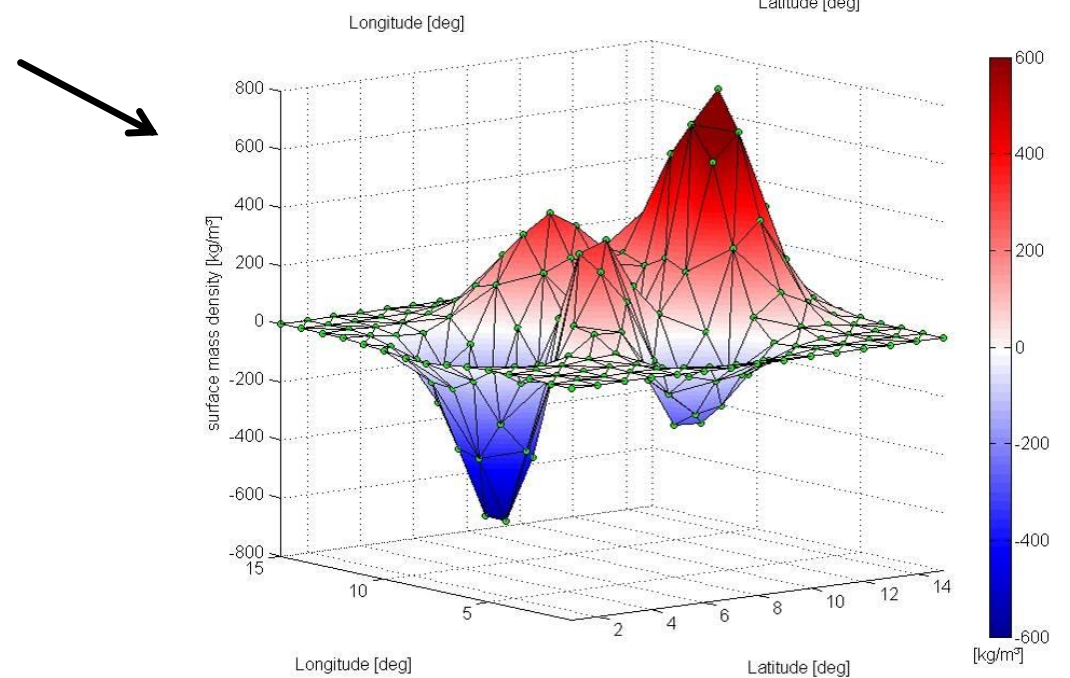

e.g. triangles 


\section{Basic principle}

Example: Consider the single layer potential

$$
V=\int_{\Omega} \frac{\sigma\left(\vec{X}_{Q}\right)}{\left\|\vec{X}-\vec{X}_{Q}\right\|} d \vec{X}_{Q}
$$

Separation of the surface into elements:

$$
\Omega=\sum_{i=1}^{N} \Omega_{i}
$$

Assumption:

$$
\begin{gathered}
\vec{X}=\sum_{k=1}^{K} \Phi_{i, k}^{\vec{X}} \vec{X}_{i, k} \\
\sigma_{i}\left(\vec{X}_{Q}\right)=\sum_{k=1}^{K} \Phi_{i, k}^{\sigma} \sigma_{i, k}\left(\vec{X}_{i, k}\right)
\end{gathered}
$$

The boundary elements are called isoparametric if $\Phi_{i, k}^{\vec{X}}=\Phi_{i, k}^{\sigma}$ 


\section{Basic principle}

Example: Consider the single layer potential

Including transformation to the normal triangle and spherical integration

$$
V=\frac{G R^{2}}{4 \pi} \sum_{i=1}^{N} \sum_{k=1}^{K} \sigma_{i, k} \int_{-1}^{1} \int_{-1}^{-\xi} \frac{J_{i}(\xi, \eta) \cdot \Phi_{i, k}(\xi, \eta) \cdot \cos \phi(\xi, \eta)}{\|\vec{X}(r, \phi, \lambda)-\vec{X}(R, \phi(\xi, \eta), \lambda(\xi, \eta))\|} d \eta d \xi
$$

Integration by Gaussian quadrature

$$
\int_{-1}^{1} \int_{-1}^{-\xi} \ldots d \eta d \xi \Rightarrow \sum_{l=1}^{L} \sum_{m=1}^{L} w_{l} w_{m} \ldots \quad \text { with } w_{l} w_{m}=0 \text { for } m>l
$$

Integration is exact for a polynomial of order $2 L$. 


\section{Mathematical properties}

Base functions are

- strictly space-limited (i.e. band-unlimited)

- compact

- continuous but not differentiable at the edges

- singular if the point of interest lies inside or on the corner of the element

- Weak singularity for potential $\left(\frac{1}{r}\right)$

- Strong singularity for gradient $\left(\frac{1}{r^{3}}\right)$ 


\section{Base function}

Example: linear trianale
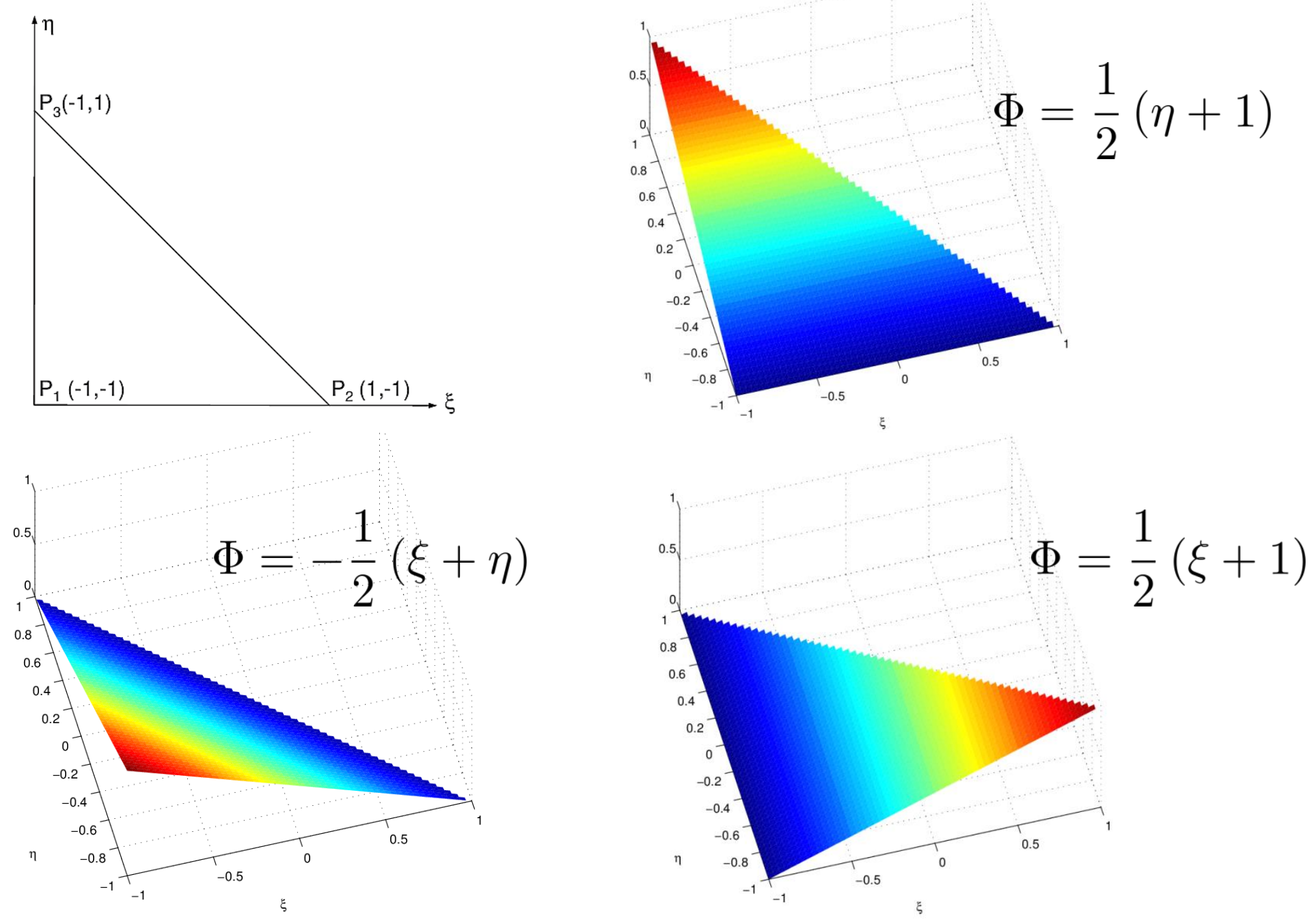


\section{Other base functions}

six node triangle

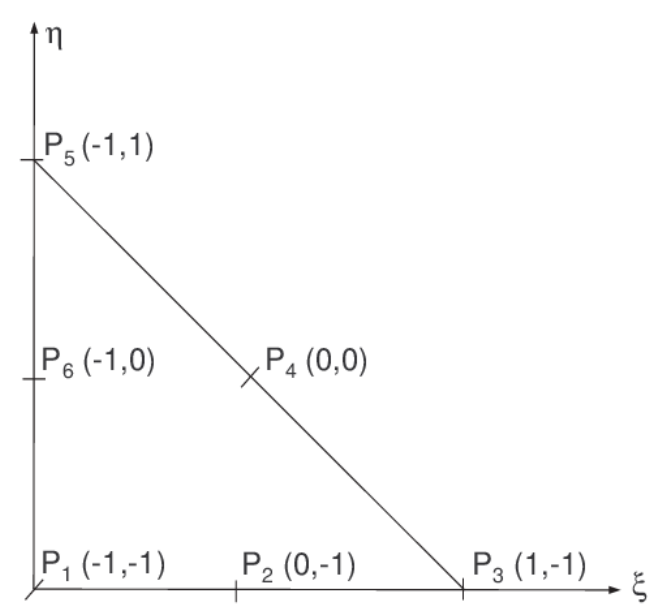

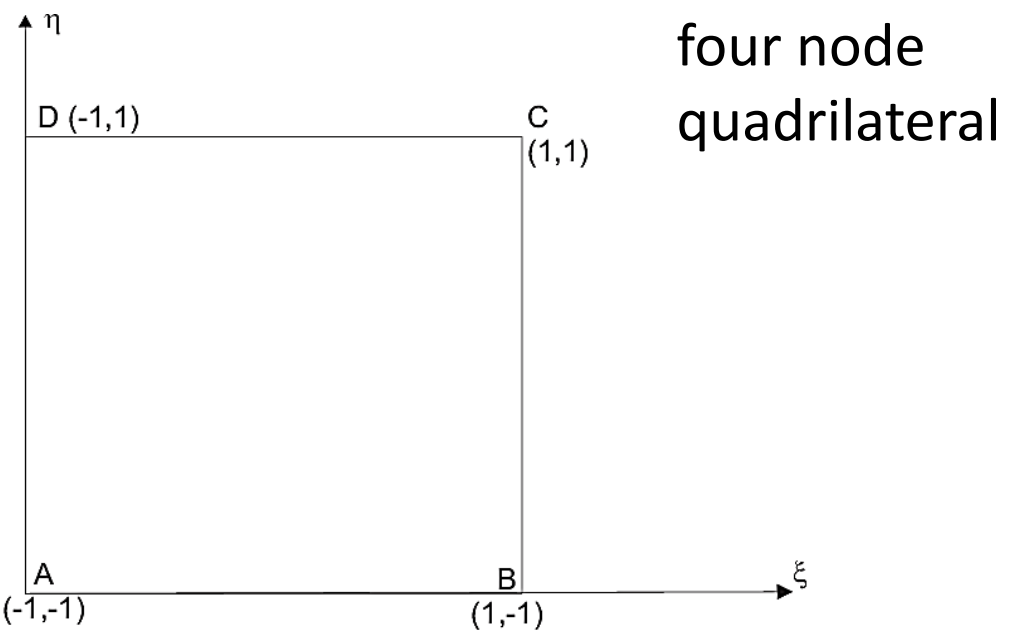

eight node quadrilateral:

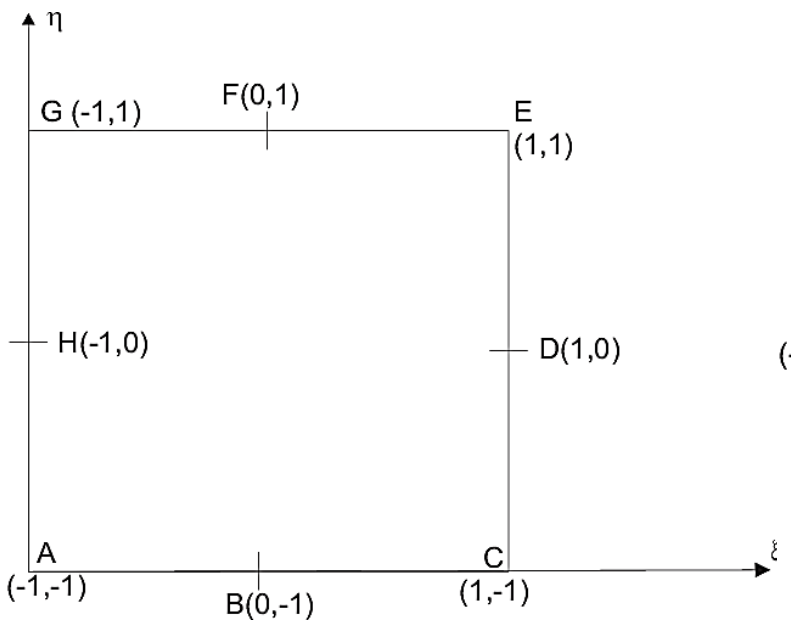

nine node quadrilateral:

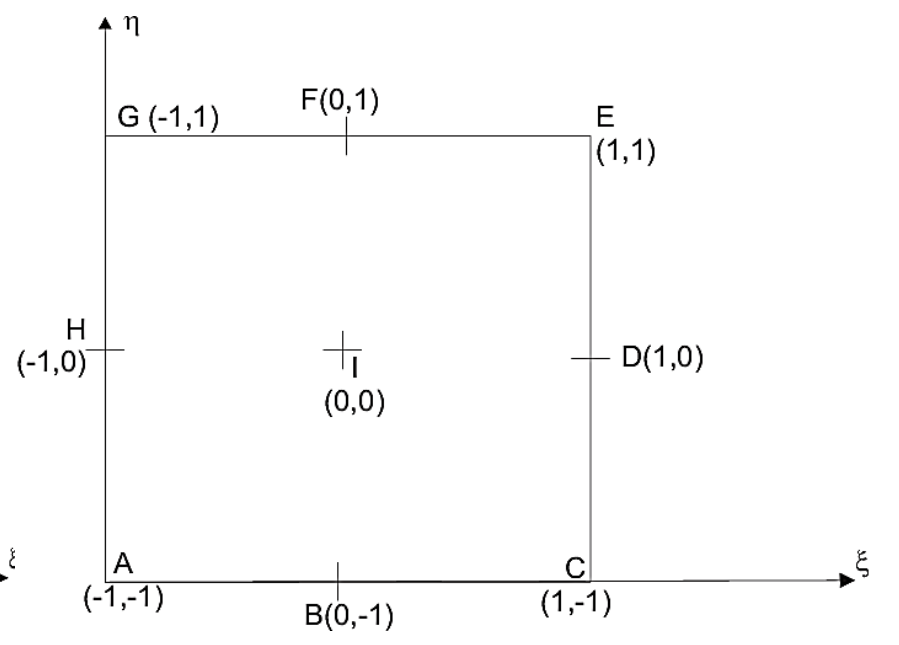

infinite quadrilateral:

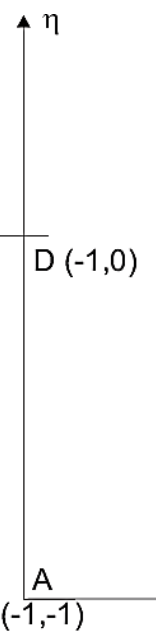




\section{Solution approach}

- Search for maxima and minima of an a priori field

- Triangulation by Delaunay tessellation

- Least-squares adjustment

- brute-force

- assembly of the normal matrix (singularity!)

- full consideration of the stochastic information

- No regularization

- objective: avoid regularization by proper grid

- iterative search for vertices 


\section{Simulation study}

\section{Closed loop simulation: noisefree and $\mathrm{h}=0 \mathrm{~km}$}
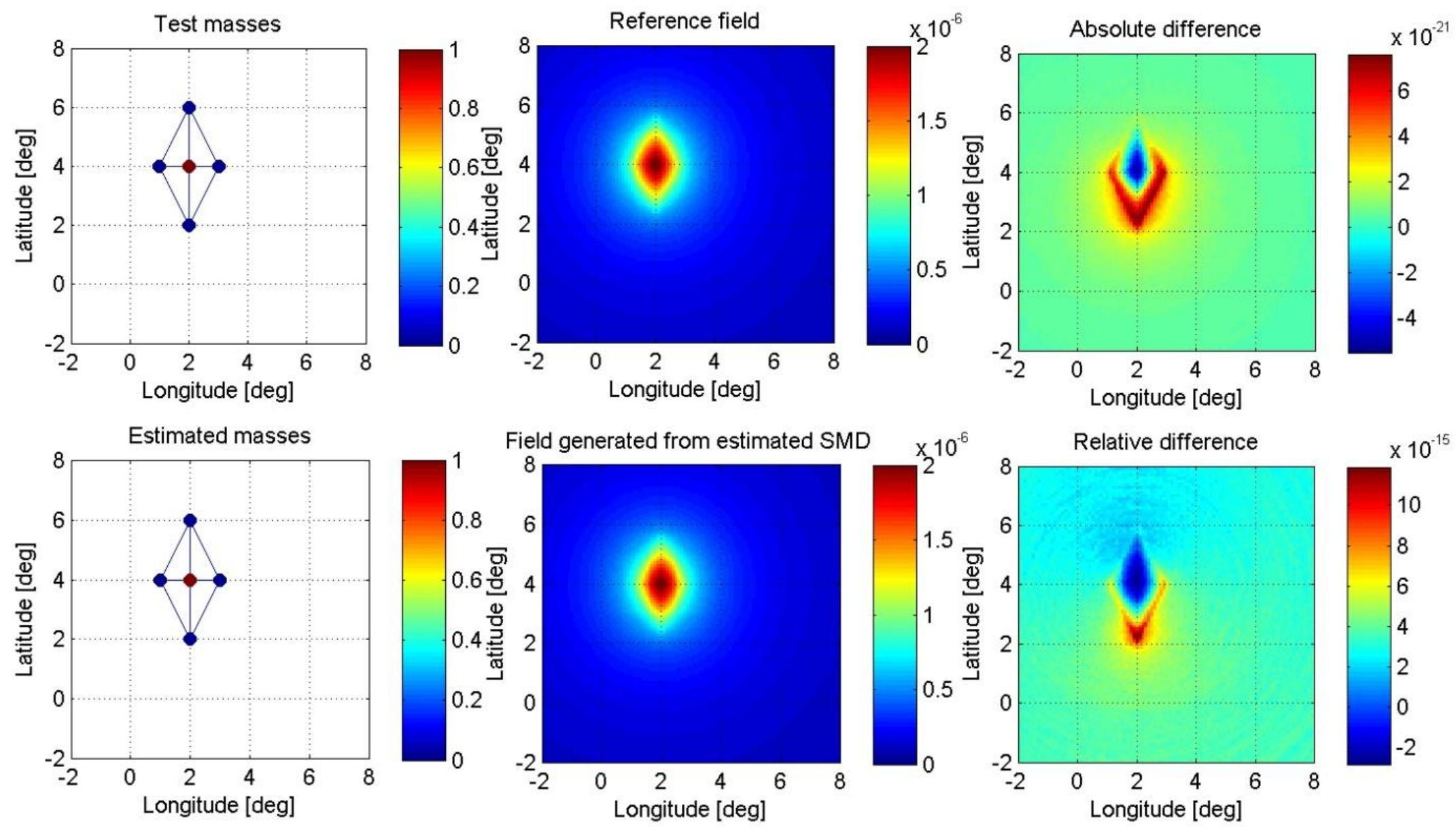

\section{RegGrav Workshop}

Feb. 23-24, 2012, Munich 


\section{Simulation study}

\section{Closed loop simulation: noise $=1 \%$ and $\mathrm{h}=400 \mathrm{~km}$}
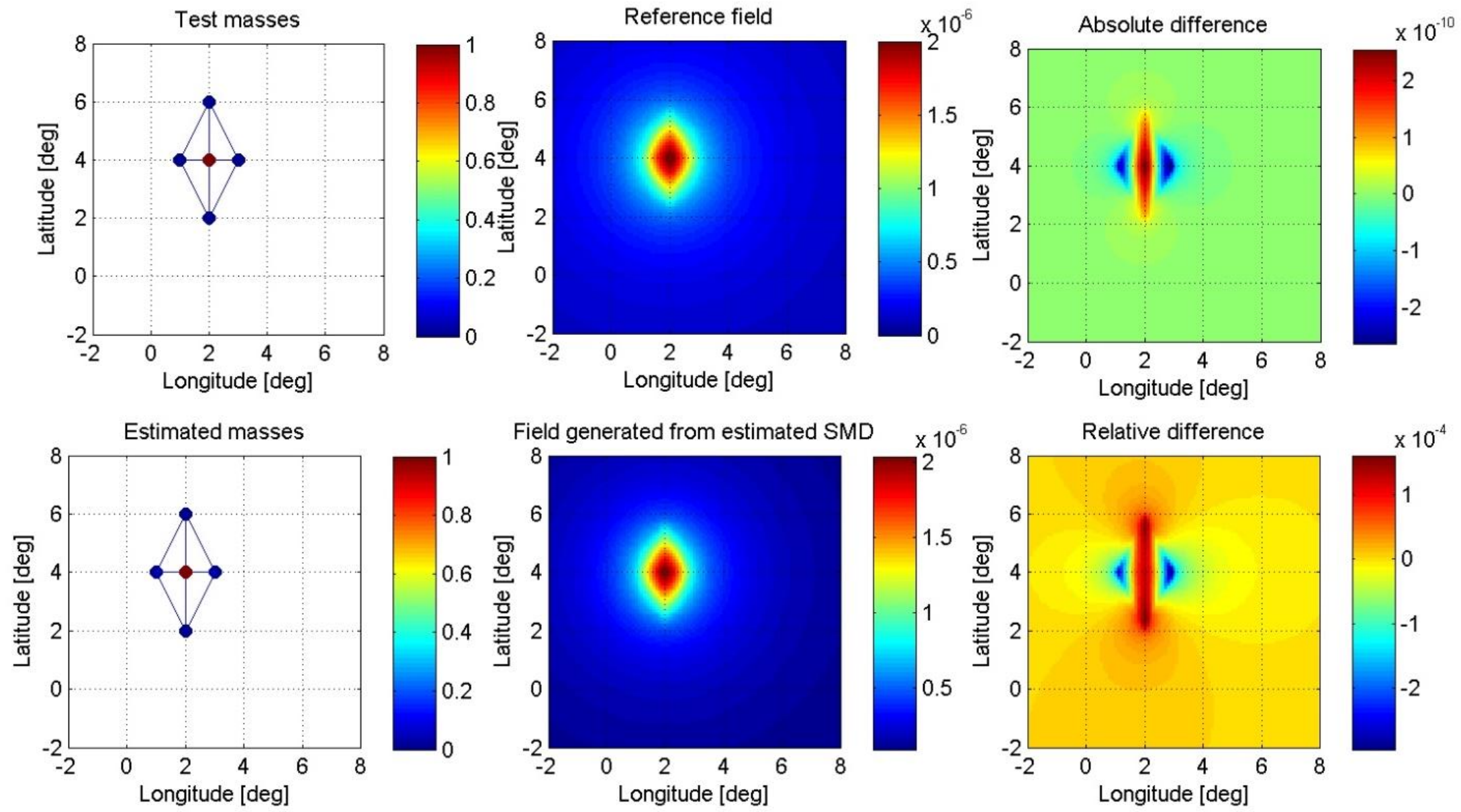

RegGrav Workshop

Feb. 23-24, 2012, Munich 


\section{Simulation study}
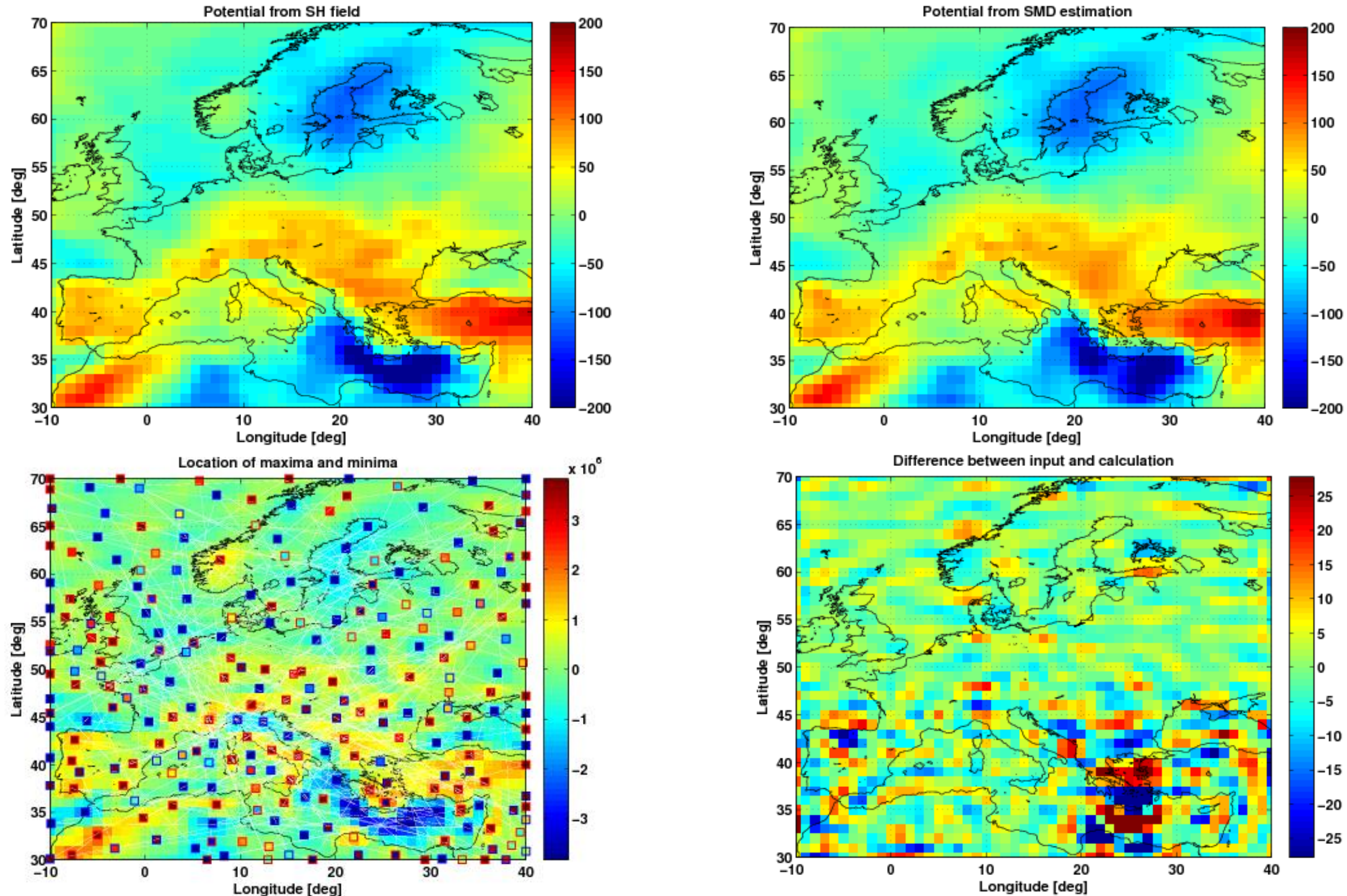

RegGrav Workshop

Feb. 23-24, 2012, Munich 


\section{Open questions}

- Optimal placement of the surface masses

- considering other quantities, e.g. curvature

- considering quadratic base elements

- Optimal tessellation using also combinations of elements

- Edge effect

- RCR technique

- Infinite elements?

- Regularization

- avoidable by choosing the proper grid (?) 


\section{ICCT study group JSG 0.6}

\section{Applicability of current GRACE solution strategies to the next generation of inter-satellite range observations}

Chairs: Matthias Weigelt, Co-Chair Adrian Jäggi

In collaboration with JSG 0.3

Comparison of methodologies for regional gravity field modelling Chairs: M. Schmidt, Co-Chair: Ch. Gerlach 


\section{Background}

$\ddot{\rho}-\frac{1}{\rho}\left(\vec{X}_{A B} \cdot \vec{X}_{A B}-\dot{\rho}^{2}\right)=\nabla V_{A B} \cdot \vec{e}_{A B}$

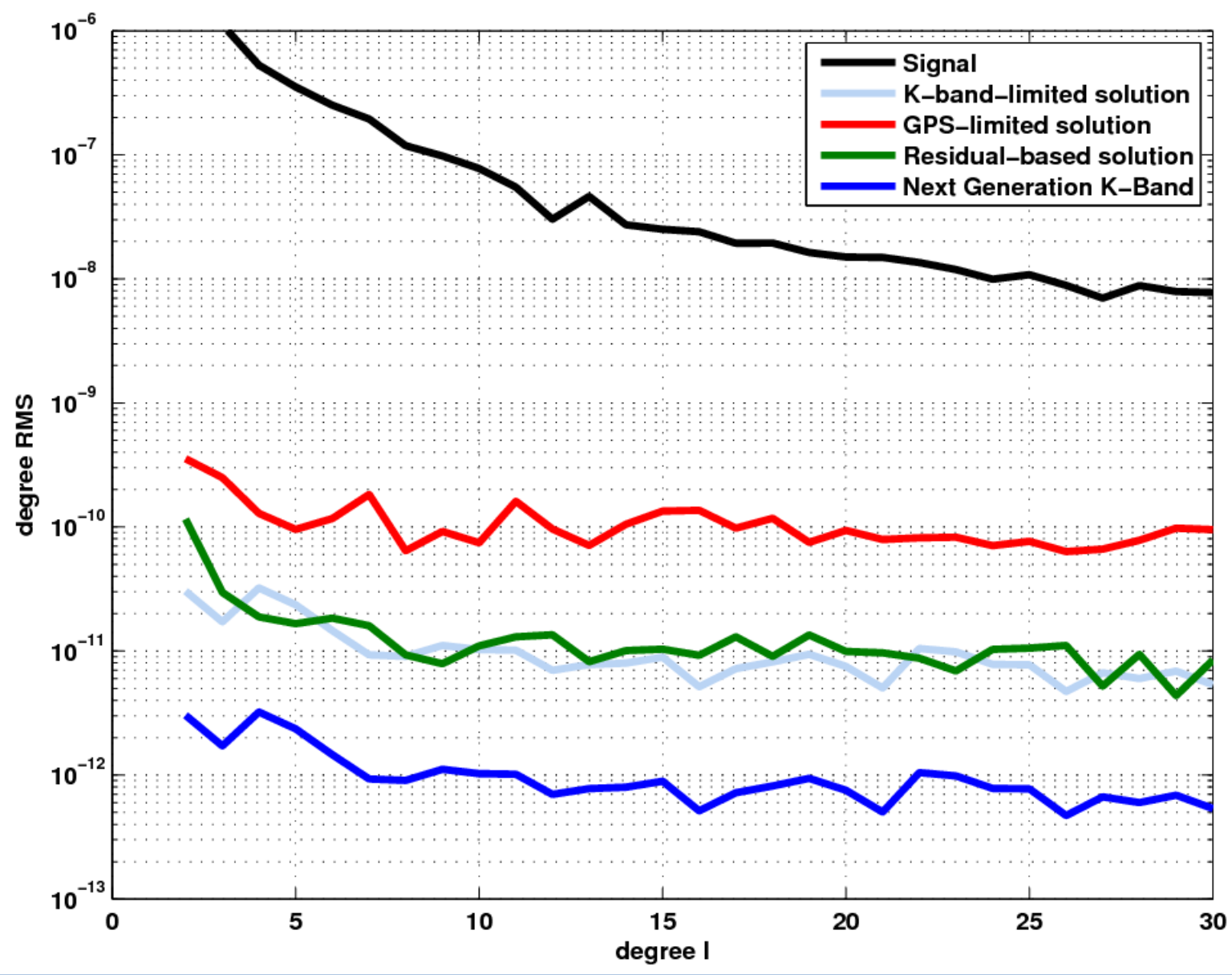

RegGrav Workshop

Feb. 23-24, 2012, Munich 


\section{Approaches}

\section{Variational equations}

\section{$\dot{\rho} \quad$ Classical \\ (Reigber 1989, Tapley 2004)}

Celestial mechanics approach

$\rho, \dot{\rho}, \Delta \rho \quad$ (Beutler et al. 2010, Jäggi 2007)

$$
\rho, \dot{\rho} \quad \begin{gathered}
\text { Short-arc method } \\
\text { (Mayer-Gürr 2006) }
\end{gathered}
$$

\section{In-situ observations}

\begin{tabular}{|cc|}
\hline $\begin{array}{c}\text { Energy Integral } \\
\text { (Han 2003, Ramillien et al. 2010) }\end{array}$ & $\dot{\rho}$ \\
\hline \hline $\begin{array}{cc}\text { Differential gravimetry } \\
\text { (Liu 2010) }\end{array}$ & $\ddot{\rho}$ \\
\hline $\begin{array}{cc}\text { LoS Gradiometry } \\
\text { (Keller and Sharifi 2005) }\end{array}$ & $\underline{\rho}$ \\
\hline
\end{tabular}

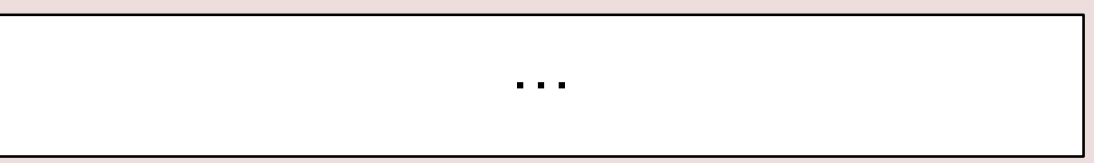

RegGrav Workshop

Feb. 23-24, 2012, Munich 


\section{Objectives}

The objectives of the study group are therefore to:

- investigate each solution strategy, identify approximations and linearizations and test them for their permissibility to the next generation of inter-satellite range observations,

- identify limitations or the necessity for additional and/or more accurate measurements,

- quantify the sensitivity to error sources, e.g. in tidal or nongravitational force modeling,

- investigate the interaction with global and local modeling,

- extend the applicability to planetary satellite mission, e.g. GRAIL

- establish a platform for the discussion and in-depth understanding of each approach and provide documentation. 